\title{
CONSERVATION LAWS IN VISCOELASTICITY
}

\author{
BY \\ GIACOMO CAVIGLIA AND ANGELO MORRO \\ Lniversità degli Studi di Genova, Genoa, Italy
}

\begin{abstract}
The evolution equations of a linear viscoelastic solid are written in terms of the Laplace transform of the displacement field. A corresponding reformulation of the condition of vanishing divergence for vector fields is then proposed and, through a systematic procedure, an explicit representation for a very large family of such conserved vectors is derived. As an application it is shown how a suitable choice of the admissible parameters leads to specific conservation laws which involve spatial means of linear momentum, angular momentum, stress, and displacement, in terms of the known body force, and initial and boundary data. As a further application a Betti-type reciprocity relation is derived. The connection with Noether's approach to conservation laws is also discussed.
\end{abstract}

1. Introduction. Consider a system of partial differential equations of the form

$$
\Delta_{i}\left(x_{\alpha}, \phi_{j}, \phi_{j \alpha}, \phi_{j \alpha \beta}\right)=0,
$$

in the unknown functions $\phi_{j}\left(x_{\alpha}\right)$, where Greek (Latin) indices vary from 1 to $n$ $(m), \phi_{j \kappa}=\partial \phi_{j} / \partial x_{\alpha}, \phi_{j \kappa \beta}=\partial^{2} \phi_{j} / \partial x_{\alpha \gamma} \partial x_{\beta}$, and the $\Delta_{i}$ 's are sufficiently regular functions of their arguments. To save writing we omit all hypotheses concerning continuity, differentiability, etc., whenever it is clear from the context what these ought to be.

Suppose $\mathbf{J}$ is a vector function of components $J_{\alpha}\left(x_{\beta}, \phi_{j}, \phi_{j \beta}\right)$. A conservation law for the system (1.1) is an equation of the form

$$
D_{\alpha} J_{\alpha} \doteq 0,
$$

in the unknowns $J_{c}$, where the symbol $\doteq$ means that equality holds on solutions to (1.1), $D_{\alpha}$ denotes the total derivative with respect to $x_{\alpha}$, that is

$$
D_{\kappa}=\frac{\partial}{\partial x_{\alpha}}+\phi_{j \alpha} \frac{\partial}{\partial \phi_{j}}+\phi_{j \beta \kappa} \frac{\partial}{\partial \phi_{j \beta}},
$$

and the summation convention on repeated indices is in force. Whenever (1.2) holds the vector $\mathbf{J}$ is said to be conserved or divergence free. The knowledge of conservation laws for systems of partial differential equations allows one to gain useful Received July 19, 1989. 
information on the properties of the solutions. That is why the generation of conservation laws is a topic which has always attracted a deep attention [1].

The search for conservation laws is usually performed up to trivialities, which means that none of the following conditions holds: (i) $\mathbf{J}$ vanishes identically on the solutions to $(1.1)$ or (ii) $D_{\alpha} J_{\alpha}=0$ for every set of functions $\phi_{j}$ regardless of whether they solve the system (1.1) [1]. No matter which of the two conditions may occur, trivial conservation laws do not have any physical significance nor do they provide any information about the properties of the solution to the given system of differential equations. That is why we are interested only in nontrivial conservation laws. In addition we regard as equivalent two conservation laws if they make a suitable linear combination, with constant coefficients, a trivial conservation law and we look for independent, that is nonequivalent, conserved vectors.

Under the assumption that the system (1.1) is self-adjoint and thus may be derived from a variational principle [2], Noether's theorem provides a straightforward means for associating conservation laws to each one-parameter variational symmetry group $[1,3,4]$. Recent investigations aiming at specific applications have led to rather general formulations of Noether's theorem which allow, e.g., dealing with conserved vectors depending on higher-order derivatives of the $\phi_{j}$ 's $[5,6]$. Besides these improvements, the recent literature has also exhibited methods for the determination of conserved vectors for non-self-adjoint systems through the solution of the so-called perturbation equation for the system (1.1) and the use of Green's theorem [7, 8].

The direct approach, which consists in finding the general solution to (1.2) under the constraint (1.1), has been used only in a few cases (see, e.g., [9]), perhaps because of difficulties in giving the physical interpretation of $\mathbf{J}$. To our mind, however, this method is very efficient and can be used profitably even when a variational formulation is allowed, as the present paper will show. Indeed, we think that this work is a remarkable step towards a general approach to the generation of conservation laws for systems with memory.

To fix ideas, we look at the dynamics of (linear) viscoelastic bodies [10], which constitute the simplest model for deformable solids when dissipation and hereditary effects are incorporated. The corresponding mathematical scheme involves a system of integro-differential equations, with the integrals reflecting the memory effects. The occurrence of integro-differential equations strongly suggests that we apply the Laplace transformation, so that a suitable reformulation of (1.2) is solved in the space of transformed variables. By means of this procedure we are able to generate previous results (given in $[11,12]$ ) and to provide rather general conserved vectors. Particular examples show how these vectors lead to conservation laws which involve initial-boundary data and provide estimates for the unknown displacement and its time derivative.

The conservation laws turn out to be nonlocal in that they involve convolutions. Families of nonlocal conservation laws, which however do not involve convolutions, have already been derived (e.g., in [13]; cf. also [14]) in connection with nongeometric symmetries of the Maxwell equations. It is of interest to reinvestigate these extensions of the concept of local conservation law and possibly to connect the unknown function to the initial and boundary data. This connection is provided in the present paper. 
2. Preliminaries on viscoelasticity. Let $\mathscr{D}$ be a closed, bounded regular region in the three-dimensional euclidean point space $\mathscr{E}$ occupied by a homogeneous viscoelastic solid in its stress-free configuration. Let $\mathscr{V}$ be the vector space associated with $\mathscr{E}$ and Lin be the space of linear transformations $\mathbf{L}(\mathscr{V}, \mathscr{V})$ from $\mathscr{V}$ into $\mathscr{V}$ (or second-order tensors).

The motion of the solid is described by the function $\mathbf{y}(\mathbf{x}, t)$ giving the position vector $\mathbf{y}$ at time $t \in \mathscr{R}$ of the particle located at $\mathbf{x}$; henceforth $\mathbf{x} \in \mathscr{D}$. It is convenient to introduce the displacement vector field $\mathbf{u}$ associated with the motion, $\mathbf{u}: \mathscr{D} \times \mathscr{R} \mapsto \mathscr{V}$, defined as

$$
\mathbf{u}(\mathbf{x}, t)=\mathbf{y}(\mathbf{x}, t)-\mathbf{x} .
$$

The unknowns $\phi_{i}$ are identified with the Cartesian components $u_{i}$ of the vector $\mathbf{u}$, so that latin indices run from 1 to 3 . We consider independent variables $x_{c}$, with $\alpha=1,2,3,4$, and we let $x_{1}, x_{2}, x_{3}$, and $x_{4}$ denote the Cartesian coordinates and the time $t$, respectively. Accordingly, e.g., $u_{i j}$ denotes $\partial u_{i} / \partial x_{j}$ and we set for convenience $\dot{u}_{i}=u_{i t}$, that is, a superimposed dot denotes the time derivative. Henceforth the direct tensor notation is used when convenient. A dot "." denotes contraction or inner product.

To arrive at the correspondent of the system (1.1) we define the strain tensor $\mathbf{E}: \mathscr{D} \times \mathscr{R} \mapsto$ Lin through

$$
\mathbf{E}(\mathbf{x}, t)=\operatorname{sym} \nabla \mathbf{u}(\mathbf{x}, t)
$$

where sym denotes the symmetric part and $\nabla$ stands for the gradient operator. On the assumption that the medium is in its undeformed state, and hence $\mathbf{E}=\mathbf{0}$ for all $t$ in $(0, \infty)$ we write the constitutive equations of linear viscoelasticity as [15]

$$
\mathbf{T}(\mathbf{x}, t)=\mathbf{G}(t) \mathbf{E}(\mathbf{x}, 0)+\int_{0}^{t} \mathbf{G}(t-\tau) \dot{\mathbf{E}}(\mathbf{x}, \tau) d \tau,
$$

where $\mathbf{T}$ is the stress tensor and $\mathbf{G}: \mathscr{Z} \times[0, \infty) \mapsto \mathbf{L}(\operatorname{Lin}, \mathrm{Lin})$ is the relaxation tensor satisfying the usual symmetry relations that in Cartesian components read

$$
G_{i j h k}=G_{j i h k}=G_{j i k h}=G_{k h j i} .
$$

We call viscoelastic state a triple $(\mathbf{u}, \mathbf{E}, \mathbf{T})$ on $\overline{\mathscr{D}} \times \mathscr{R}$ of displacement field u, strain tensor $\mathbf{E}$, and stress tensor $\mathbf{T}$, related by (2.1). The convolution form for the viscoelastic state equation (2.1) will be decisively advantageous in the next developments.

Letting $\rho$ be the constant mass density and $\mathbf{F}(\mathbf{x}, t)$ the body force, the equations of motion are

$$
\rho \ddot{\mathbf{u}}=\nabla \cdot \mathbf{T}+\mathbf{F},
$$

subject to the initial conditions

$$
\mathbf{u}(\mathbf{x}, 0)=\mathbf{d}(\mathbf{x}), \quad \dot{\mathbf{u}}(\mathbf{x}, 0)=\mathbf{v}(\mathbf{x}) .
$$


As discussed in [11], (2.2) and (2.3) are equivalent to the single equation

$$
\rho \mathbf{u}=g * \nabla \cdot \mathbf{T}+\mathbf{f} \text { in } \mathscr{D} \times[0, \infty),
$$

where $*$ denotes the convolution,

$$
\begin{gathered}
g(t)=t, \quad t \in[0, \infty), \\
\mathbf{f}(\mathbf{x}, t)=[g * \mathbf{F}](\mathbf{x}, t)+\rho[t \mathbf{v}(\mathbf{x})+\mathbf{d}(\mathbf{x})], \quad t \in[0, \infty) .
\end{gathered}
$$

We now apply the Laplace transform operator $\mathscr{L}: \mathscr{L}\{h(\mathbf{x}, t)\}=\tilde{h}(\mathbf{x}, p)$ to obtain

$$
\rho \tilde{\mathbf{u}}(\mathbf{x}, p)=\frac{1}{p^{2}} \mathscr{L}\{\nabla \cdot \mathbf{T}\}+\frac{1}{p^{2}} \widetilde{\mathbf{F}}(\mathbf{x}, p)+\rho\left[\frac{1}{p^{2}} \mathbf{v}(\mathbf{x})+\frac{1}{p} \mathbf{d}(\mathbf{x})\right] .
$$

Hence it follows from (2.1) that

$$
\mathscr{L}\{\nabla \cdot \mathbf{T}\}=\nabla \cdot[p \tilde{\mathbf{G}}(p) \nabla \tilde{\mathbf{u}}] .
$$

Substitution into (2.6) yields the vector form of the transformed equation of motion; the explicit expression of its $i$-th component reads

$$
\rho \tilde{u}_{i}=\frac{1}{2 p} \tilde{A}_{i j k l} \tilde{u}_{l k j}+\tilde{f}_{i}
$$

where the tensor $\widetilde{\mathbf{A}}$ is defined by

$$
\tilde{A}_{i j k l}=\tilde{G}_{i j k l}+\tilde{G}_{i k j l} .
$$

The vector $\tilde{\mathbf{f}}$ is the Laplace transform of (2.6), namely

$$
\tilde{\mathbf{f}}=\frac{1}{p^{2}} \widetilde{\mathbf{F}}+\rho\left(\frac{\mathbf{v}}{p^{2}}+\frac{\mathbf{d}}{p}\right),
$$

and the dependence on the parameter $p$ is henceforth understood and not written. Incidentally, we observe that, by mistake, in [12] Eq. (2.8) has been written with the additive term $\nabla \cdot(\widetilde{\mathbf{G}} \nabla \mathbf{d})$.

For later convenience we observe that in view of (2.2) and (2.9) the tensor $\tilde{\mathbf{A}}$ satisfies the symmetry properties

$$
\tilde{A}_{i j k l}=\tilde{A}_{i k j l}=\tilde{A}_{l j k i}=\tilde{A}_{k l i j} .
$$

It is also apparent that Eq. (2.8) is the Euler-Lagrange equation corresponding to the Lagrangian density $\widehat{L}$ given by

$$
\widehat{L}=\frac{1}{2 p} \tilde{G}_{i j k l} \tilde{u}_{i j} \tilde{u}_{k l}-\tilde{f}_{i} \tilde{u}_{i}+\frac{1}{2} \rho \tilde{u}_{i} \tilde{u}_{i} .
$$

3. General form of conservation laws. According to our notation, a conservation law is a relation of the form

$$
D_{i} J_{i}+D_{t} I \doteq 0
$$

where, for convenience, $I$ stands for $J_{t}$ and the symbol $\doteq$ means that equality holds along solutions to (2.3). In this framework $I$ is usually referred to as a conserved density and the associated $J_{i}$ 's identify the corresponding flux.

Owing to the structure of $(2.1)$ and (2.3), in the case of viscoelasticity $J_{i}$ and $I$ at the point $\mathbf{x}$ and time $t$ are likely to be functionals on a normed space $\mathscr{H}$ of 
histories of $\mathbf{u}$ and $\nabla \mathbf{u}$, at $\mathbf{x}$, up to time $t$. This dependence, however, would make the search for the solution $J_{i}, I$ a formidable problem. With this in mind, and taking advantage of the convolution form of (2.6), we set up an alternative characterization of conservation laws by arguing as follows.

The formal application of the Laplace transform to (3.1) yields

$$
D_{i} \tilde{J}_{i}+p \tilde{I}-I_{0} \doteq 0,
$$

where $I_{0}$ is the restriction of $I$ to $t=0$ and the field equations are now given by (2.8). Therefore, in view of the well-known properties of convolutions under Laplace transformation, we expect that in terms of $\tilde{\mathbf{u}}$ the conservation law can be given the form

$$
D_{i} \widehat{J}_{i}+p \widehat{I}-I_{0} \doteq 0
$$

with $\widehat{J}_{i}$ and $\widehat{I}$ functions of $(\mathbf{x}, \tilde{\mathbf{u}}, \nabla \tilde{\mathbf{u}}) \in \mathscr{D} \times \mathscr{V} \times \operatorname{Lin}$. In (3.2) $I_{0}$ stands for a scalar function on $\mathscr{D}$ to be determined. Of course, the Laplace variable $p$ enters $\widehat{J}_{i}$ and $\widehat{I}$ as a parameter. Explicitly, (3.2) can be written as

$$
\frac{\partial \widehat{J}_{i}}{\partial x_{i}}+\frac{\partial \widehat{J}_{i}}{\partial \tilde{u}_{j}} \tilde{u}_{j i}+\frac{\partial \widehat{J}_{i}}{\partial \tilde{u}_{j h}} \tilde{u}_{j h i}+p \widehat{I}-I_{0} \doteq 0 .
$$

Our aim is to solve $(3.3)$ for $\widehat{J}_{i}$ and $\widehat{I}$ and to determine their inverse Laplace transform, in order to find conservation laws in the physical space; in applying this procedure we will identify $I_{0}$ with the expression at $t=0$ of the inverse transform of $\widehat{I}$.

The first step in our approach yields the explicit form of the dependence of $\widehat{J}_{i}$ on $\nabla \tilde{\mathbf{u}}$. Under rather mild conditions of nondegeneracy [1], there exist three real functions $B_{i}, i=1,2,3$, on $\mathscr{D} \times \mathscr{V} \times \operatorname{Lin}$ such that the condition (3.3) is equivalent to the validity of

$$
\frac{\partial \widehat{J}_{i}}{\partial x_{i}}+\frac{\partial \widehat{J}_{i}}{\partial \tilde{u}_{j}} \tilde{u}_{j i}+\frac{\partial \widehat{J}_{i}}{\partial \tilde{u}_{j h}} \tilde{u}_{j h i}+p \widehat{I}-I_{0}=B_{k}\left(\frac{1}{p} \tilde{A}_{k i h j} \tilde{u}_{j h i}+2 \tilde{f}_{k}-2 \rho \tilde{u}_{k}\right)
$$

for arbitrary values of $\tilde{u}_{j}, \tilde{u}_{j h}, \tilde{u}_{j h i}$. The arbitrariness of $\tilde{u}_{j h i}$, along with its symmetry with respect to the indices $h$ and $i$, implies that

$$
\left(\frac{\partial \widehat{J}_{i}}{\partial \tilde{u}_{j h}}+\frac{\partial \widehat{J}_{h}}{\partial \tilde{u}_{j i}}\right)=\frac{2}{p} B_{k} \tilde{A}_{k i h j} .
$$

On regarding (3.5) as a differential equation for the unknown functions $\widehat{J}_{i}$ we may determine the general form of the dependence of $\widehat{J}_{i}$ and $B_{k}$ on $\tilde{u}_{j q}$.

The integrability conditions for (3.5) are found by observing that suitable combinations of its partial derivatives with respect to the spatial gradient of $\tilde{\mathbf{u}}$ lead to

$$
\frac{p}{2}\left(\frac{\partial^{2} \hat{J}_{i}}{\partial \tilde{u}_{j h} \partial \tilde{u}_{s q}}+\frac{\partial^{2} \widehat{J}_{i}}{\partial \tilde{u}_{j q} \partial \tilde{u}_{s h}}\right)=\tilde{A}_{k i h j} \frac{\partial B_{k}}{\partial \tilde{u}_{s q}}-\tilde{A}_{k h q s} \frac{\partial B_{k}}{\partial \tilde{u}_{j i}}+\tilde{A}_{k i q j} \frac{\partial B_{k}}{\partial \tilde{u}_{s h}} .
$$

The left-hand side is symmetric in the pairs $j, s$ and $h, q$; on the contrary, the right-hand side is symmetric with respect to the pair $h, q$ only. Hence we have to 
impose a compatibility condition, which is obtained by taking the skew part of both sides with respect to the indices $j$ and $s$. This yields

$$
\left(\tilde{A}_{k i h j} \frac{\partial B_{k}}{\partial \tilde{u}_{s q}}-\tilde{A}_{k i h s} \frac{\partial B_{k}}{\partial \tilde{u}_{j q}}\right)_{(i h q)}=0,
$$

the subscript $(i h q)$ denoting total symmetrization over $i h q$.

Equation (3.6) is written in the form of a homogeneous linear system in the unknowns $\partial B_{k} / \partial \tilde{u}_{s q}$, which is to hold in correspondence with any choice of the coefficients $\tilde{A}_{k i h j}$ obeying the symmetry requirement $(2.11)$. Suppose we set

$$
\tilde{A}_{k i h j}=\lambda \delta_{k j} \delta_{i h}+\mu\left(\delta_{k h} \delta_{i j}+\delta_{k i} \delta_{h j}\right),
$$

where $\lambda$ and $\mu$ are nonvanishing constants with $\lambda \neq \mu$. Substitution into (3.6) leads to the necessary condition

$$
\frac{\partial B_{k}}{\partial \tilde{u}_{s q}}=\delta_{k s} \alpha_{q},
$$

where $\alpha_{q}$ is defined in $\mathscr{Z} \times \mathscr{V} \times$ Lin. This condition is also sufficient, in the sense that upon substitution of (3.7) into (3.6) it is found that the expression within round brackets vanishes identically in view of (2.11).

Equation (3.7) yields the expression of $B_{k}$. By the integrability conditions of (3.7) we obtain that $\alpha_{q}$ cannot depend on $\nabla \tilde{\mathbf{u}}$, whence it follows that $B_{k}$ may be represented as

$$
B_{k}=\frac{1}{2}\left[\tilde{u}_{k q}\left(\alpha_{q}+\bar{\alpha}_{q}\right)+\beta_{k}+\bar{\beta}_{k}\right]
$$

with $\alpha_{q}, \beta_{k}, \bar{\alpha}_{q}, \bar{\beta}_{k}$, as functions of $\mathbf{x}$, $\tilde{\mathbf{u}}$, and the parameter $p, \bar{\alpha}_{q}$ and $\bar{\beta}_{k}$ having been introduced for convenience.

Upon substitution of (3.8) into (3.5) we conclude that the most general solution to $(3.5)$ can be written as

$$
\begin{aligned}
\widehat{J}_{i}=\frac{1}{p}[ & \frac{1}{2}\left(\tilde{u}_{k q} \alpha_{q}+\beta_{k}\right) \tilde{A}_{k i h j} \tilde{u}_{j h}-\frac{1}{4} \alpha_{i} \tilde{A}_{j q s r} \tilde{u}_{j q} \tilde{u}_{r s} \\
& \left.+\left(\tilde{u}_{k q} \bar{\alpha}_{q}+\bar{\beta}_{k}\right) \tilde{G}_{k i h j} \tilde{u}_{j h}-\frac{1}{2} \bar{\alpha}_{i} \tilde{G}_{j q s r} \tilde{u}_{j q} \tilde{u}_{r s}\right]+\nu_{i j h} \tilde{u}_{j h}+k_{i},
\end{aligned}
$$

where $\nu_{i j h}$ and $k_{i}$ depend on $\mathbf{x}, \tilde{\mathbf{u}}$ and, in addition, $\nu_{i j h}=-\nu_{h j i}$. Comparing with (3.5), (3.8), and (3.9) it follows that (3.4) becomes

$$
\begin{aligned}
& \frac{1}{p}\left\{\frac{1}{2}\left[\tilde{u}_{k q}\left(\frac{\partial \alpha_{q}}{\partial x_{i}}+\frac{\partial \alpha_{q}}{\partial \tilde{u}_{l}} \tilde{u}_{l i}\right)+\frac{\partial \beta_{k}}{\partial x_{i}}+\frac{\partial \beta_{k}}{\partial \tilde{u}_{l}} \tilde{u}_{l i}\right] \tilde{A}_{k i h j} \tilde{u}_{j h}-\frac{1}{4}\left(\frac{\partial \alpha_{i}}{\partial x_{i}}+\frac{\partial \alpha_{i}}{\partial \tilde{u}_{l}} \tilde{u}_{l i}\right)\right. \\
& \times \tilde{A}_{j q s r} \tilde{u}_{j q} \tilde{u}_{r s}+\left[\tilde{u}_{k q}\left(\frac{\partial \bar{\alpha}_{q}}{\partial x_{i}}+\frac{\partial \bar{\alpha}_{q}}{\partial \tilde{u}_{l}} \tilde{u}_{l i}\right)+\frac{\partial \bar{\beta}_{k}}{\partial x_{i}}+\frac{\partial \bar{\beta}_{k}}{\partial \tilde{u}_{l}} \tilde{u}_{l i}\right] \tilde{G}_{k i h j} \tilde{u}_{j h} \\
& \left.-\frac{1}{2}\left(\frac{\partial \bar{\alpha}_{i}}{\partial x_{i}}+\frac{\partial \bar{k}_{i}}{\partial \tilde{u}_{l}} \tilde{u}_{/ i}\right) \tilde{G}_{j q s r} \tilde{u}_{j q} \tilde{u}_{r s}\right\} \\
& +\frac{\partial \nu_{i j h}}{\partial x_{i}} \tilde{u}_{j h}+\frac{\partial \nu_{i j h}}{\partial \dot{u}_{l}} \tilde{u}_{l i} \tilde{u}_{j h}+\frac{\partial k_{i}}{\partial x_{i}}+\frac{\partial k_{i}}{\partial \tilde{u}_{l}} \tilde{u}_{l i} \\
& +p \hat{I}-I_{0}+\left[\tilde{u}_{k q}\left(\alpha_{q}+\alpha_{q}\right)+\beta_{k}+\bar{\beta}_{k}\right]\left(\rho \tilde{u}_{k}-\tilde{f}_{k}\right)=0 .
\end{aligned}
$$


The next step consists in finding the form of the functions $\alpha_{q}, \beta_{k}, \bar{\alpha}_{q}, \bar{\beta}_{k}, \nu_{i j h}$, $k_{i}$, and $\hat{I}$ which satisfy (3.10). The conservation law (3.10) is in fact a family of conservation laws. Actually, it seems that in view of the arbitrariness allowed in the determination of $\hat{I}$, almost any choice of $\widehat{\mathbf{J}}$ gives rise to a conservation law. However, we have to take into account two basic facts. First, the expressions for the admissible functions should be simple enough to allow for the application of the inverse Laplace transform; in practice, this is guaranteed by taking polynomial dependences on $\tilde{\mathbf{u}}$ and its spatial derivatives. Second, the arbitrariness in the choice of $\widehat{I}$ is restricted by the requirement that the resulting conservation law is not trivial. In this connection it is worth observing that the terms which precede $p \widehat{I}$ in Eq. (3.10) cannot simply be removed by collecting them into the definition of $\hat{I}$ in order to achieve maximum generality; this would imply the appearance of a spatial divergence in the expression of $\widehat{I}$, which ultimately should lead to a trivial conservation law in the physical space.

For the sake of definiteness and to prepare the discussion of subsequent applications to physically relevant situations, henceforth we make the assumption that $\widehat{I}$ depends at most linearly on $\nabla \tilde{\mathbf{u}}$. Look at $(3.10)$ as a third-order expression in the partial derivatives $\tilde{u}_{k q}$ that must be satisfied for suitable choices of $\widehat{I}$. Then the vanishing of the contributions due to third-order and second-order terms implies that

$$
\begin{gathered}
\bar{\alpha}_{k}=2 a x_{k}+b_{k}, \quad \alpha_{k}=c x_{k}+e_{k}, \\
\bar{\beta}_{k}=a \tilde{u}_{k}+\psi_{k}, \quad \beta_{k}=\frac{1}{2} c \tilde{u}_{k}+\phi_{k}, \\
\nu_{i j h}=\frac{\partial z_{i h}}{\partial \tilde{u}_{j}},
\end{gathered}
$$

where $a, b_{k}, c, e_{k}$ are only allowed to depend on the parameter $p ; z_{\text {ih }}=$ $z_{i h}(\tilde{\mathbf{x}}, \tilde{\mathbf{u}}, p)$, with $z_{i h}=-z_{h i}$, and $\phi_{k}=\phi_{k}(\mathbf{x}, p), \psi_{k}=\psi_{k}(\mathbf{x}, p)$. As a consequence (3.10) reduces to

$$
\begin{aligned}
& \frac{1}{p}\left(\frac{1}{2} \frac{\partial \phi_{k}}{\partial x_{i}} \tilde{A}_{k i h j} \tilde{u}_{j h}+\frac{\partial \psi_{k}}{\partial x_{i}} \tilde{G}_{k i h j} \tilde{u}_{j h}\right)+\frac{\partial^{2} z_{i h}}{\partial \tilde{u}_{j} \partial x_{i}} \tilde{u}_{j h}+\frac{\partial k_{i}}{\partial x_{i}}+\frac{\partial k_{i}}{\partial \tilde{u}_{l}} \tilde{u}_{l i} \\
& \quad+p \widehat{I}-I_{0}+\left\{\tilde{u}_{k q}\left[(2 a+c) x_{q}+b_{q}+e_{q}\right]+\left(a+\frac{1}{2} c\right) \tilde{u}_{k}+\phi_{k}+\psi_{k}\right\} \\
& \quad \times\left(\rho \tilde{u}_{k}-\tilde{f}_{k}\right)=0
\end{aligned}
$$

and $\widehat{J}_{i}$ takes the form

$$
\begin{aligned}
\widehat{J}_{i}=\frac{1}{p}\left[\frac{1}{2}\left(\tilde{u}_{k q} \alpha_{q}+\beta_{k}\right) \tilde{A}_{k i h j} \tilde{u}_{j h}+\left(\tilde{u}_{k q} \bar{\alpha}_{q}+\bar{\beta}_{k}\right) \tilde{G}_{k i h j} \tilde{u}_{j h}\right. \\
\left.-\frac{1}{4} \alpha_{i} \tilde{A}_{j q s r} \tilde{u}_{j q} \tilde{u}_{r s}-\frac{1}{2} \bar{\alpha}_{i} \tilde{G}_{j q s r} \tilde{u}_{j q} \tilde{u}_{r s}\right]+\frac{\partial z_{i h}}{\partial \tilde{u}_{j}} \tilde{u}_{j h}+k_{i} .
\end{aligned}
$$


4. Explicit structure of some conservation laws. To obtain simple but physically significant examples we consider the particular case when $a, c, b_{q}, e_{q}, \phi_{k}$, and $z_{i h}$ vanish. The expressions (3.12) and (3.13) reduce to

$$
\frac{1}{p} \frac{\partial \psi_{k}}{\partial x_{i}} \widetilde{G}_{k i h j} \tilde{u}_{j h}+\frac{\partial k_{i}}{\partial x_{i}}+\frac{\partial k_{i}}{\partial \tilde{u}_{h}} \tilde{u}_{h i}+p \widehat{I}-I_{0}+\psi_{k}\left(\rho \tilde{u}_{k}-\tilde{f}_{k}\right)=0
$$

and

$$
\widehat{J}_{i}=\frac{1}{p} \psi_{k} \widetilde{G}_{k i h j} \tilde{u}_{j h}+k_{i} .
$$

Among the conservation laws are the balance laws of linear and angular momentum. With the purpose of obtaining these laws we choose $\widehat{I}$ as

$$
\widehat{I}=-\frac{1}{p} \psi_{k} \rho \tilde{u}_{k} .
$$

i) Balance laws. Now we show in detail how the balance laws follow. By (4.3), Eq. (4.1) reduces to

$$
\frac{1}{p} \frac{\partial \psi_{k}}{\partial x_{i}} \tilde{G}_{k i h j} \tilde{u}_{j h}+\frac{\partial k_{h}}{\partial x_{h}}+\frac{\partial k_{h}}{\partial \tilde{u}_{j}} \tilde{u}_{j h}-I_{0}-\psi_{k} \tilde{f}_{k}=0 .
$$

The arbitrariness of $\tilde{u}_{j h}$ gives

$$
\frac{1}{p} \frac{\partial \psi_{k}}{\partial x_{i}} \widetilde{G}_{k i h j}+\frac{\partial k_{h}}{\partial \tilde{u}_{j}}=0
$$

whence

$$
k_{h}=-\frac{1}{p} \frac{\partial \psi_{k}}{\partial x_{i}} \widetilde{G}_{k i h j} \tilde{u}_{j}+\hat{\sigma}_{h},
$$

where $\hat{\sigma}_{h}$ is a function of $\mathbf{x}$ parameterized by $p$. Meanwhile (4.4) becomes

$$
\frac{\partial k_{h}}{\partial x_{h}}-I_{0}-\psi_{k} \tilde{f}_{k}=0
$$

Letting $w_{k}$ be the inverse Laplace transform of $\tilde{w}_{k}:=\psi_{k} / p^{2}$, by (4.3) we have

$$
I=-w_{k} * \rho \dot{u}_{k}-\rho w_{k} d_{k}
$$

and

$$
I_{0}=-\rho w_{k}(0) d_{k},
$$

where $w_{k}(0)$ denotes the restriction to $t=0$ of $w_{k}$. Substitution of $k_{h}$ from (4.5) into (4.6) yields

$$
-\frac{1}{p} \frac{\partial^{2} \psi_{k}}{\partial x_{h} \partial x_{i}} \tilde{G}_{k i h j} \tilde{u}_{j}+\frac{\partial \hat{\sigma}_{h}}{\partial x_{h}}+\rho w_{k}(0) d_{k}-\psi_{k} \tilde{f}_{k}=0,
$$

where only the first term depends on $\tilde{u}_{j}$. Hence

$$
\frac{\partial^{2} \psi_{k}}{\partial x_{h} \partial x_{i}}=0
$$


The functions $\hat{\sigma}_{h}$ are then characterized through the divergence $\partial \hat{\sigma}_{h} / \partial x_{h}$ by

$$
\frac{\partial \hat{\sigma}_{h}}{\partial x_{h}}=-\rho w_{k}(0) d_{k}+\psi_{k} \tilde{f}_{k} .
$$

By (4.8) we can write

$$
w_{k}=\lambda_{k}+\left(\chi_{k h}+\omega_{k h}\right) x_{h}
$$

where $\lambda_{k}, \chi_{k h}$, and $\omega_{k h}$ are arbitrary functions of $t$ with $\chi_{k h}=\chi_{h k}, \omega_{k h}=-\omega_{h k}$. Meanwhile (4.9) can be written as

$$
\frac{\partial \hat{\sigma}_{h}}{\partial x_{h}}=\rho\left[p \tilde{w}_{k}-w_{k}(0)\right] d_{k}+\tilde{w}_{k} \widetilde{F}_{k}+\rho \tilde{w}_{k} v_{k},
$$

whence we get the divergence of $\sigma_{h}=\mathscr{L}^{-1}\left(\hat{\sigma}_{h}\right)$ as

$$
\frac{\partial \sigma_{h}}{\partial x_{h}}=\rho \dot{w}_{k} d_{k}+w_{k} * F_{k}+\rho w_{k} v_{k} .
$$

The inverse Laplace transform of (4.2) and account of (2.1) and (4.5) yield

$$
J_{i}=w_{k} * T_{k i}+\chi_{k l} *\left(G_{k l h j} * \dot{u}_{j}+G_{k l h j} d_{j}\right)+\sigma_{i} .
$$

The functions (4.7) and (4.12) provide the desired conservation law.

To get a physically more suggestive form of the balance equation we consider the global counterpart. By (3.1) and (4.12), integration over $\mathscr{D} \times[0, t)$ gives

$$
V\left(\bar{I}-\bar{I}_{0}\right)=-\int_{0}^{t} \int_{\mathscr{Z}}\left[\chi_{k l} * T_{k l}+\frac{\partial \sigma_{i}}{\partial x_{i}}\right] d x d t^{\prime}-\int_{0}^{t} \int_{\partial \mathscr{Z}} w_{k} * T_{k i} n_{i} d a d t^{\prime}
$$

where $V$ is the volume of $\mathscr{D}$, a superposed bar denotes the spatial mean in $\mathscr{D}, \mathbf{n}$ is the outward normal, $d x$ is the volume element and $d a$ is the surface element. Account of (4.11) and some rearrangement yield

$$
V \overline{w_{k} * \rho \dot{u}_{k}}-V \int_{0}^{t} \overline{\chi_{k l} * T_{k l}} d t^{\prime}=\int_{0}^{t}\left(\overline{w_{k} * F_{k}}+\rho \overline{w_{k} v_{k}}\right) d t^{\prime}+\int_{0}^{t} \int_{\partial \mathscr{Z}} w_{k} * T_{k i} n_{i} d a d t^{\prime} .
$$

The arbitrariness of $\lambda_{k}, \chi_{k l}, \omega_{k l}$ provides three separate conservation laws, namely

$$
\begin{gathered}
V(\lambda * \rho \overline{\mathbf{u}})(t)=V \int_{0}^{t}(\lambda * \overline{\mathbf{F}}+\rho \lambda \overline{\mathbf{v}}) d t^{\prime}+\int_{0}^{t} \lambda * \int_{\partial Z^{\prime}} \mathbf{T n} d a d t^{\prime} \\
V[\chi * \operatorname{sym}(\overline{\mathbf{x} \otimes \rho \dot{\mathbf{u}}})](t)+V \int_{0}^{t}(\chi * \overline{\mathbf{T}}) d t^{\prime}=V \int_{0}^{t}[\chi * \operatorname{sym}(\overline{\mathbf{x} \otimes \mathbf{F}})+\chi \operatorname{sym}(\overline{\mathbf{x} \otimes \rho \mathbf{v}})] d t^{\prime} \\
+\int_{0}^{t} \chi * \int_{\partial \mathscr{Z}} \operatorname{sym}(\mathbf{x} \otimes \mathbf{T n}) d a d t^{\prime},
\end{gathered}
$$$$
V[\omega *(\overline{\mathbf{x} \times \rho \dot{\mathbf{u}}})](t)=V \int_{0}^{t}(\omega * \overline{\mathbf{x} \times \mathbf{F}}+\omega * \overline{\mathbf{x} \times \rho \mathbf{v}}) d t^{\prime}+\int_{0}^{t} \omega * \int_{\partial \mathscr{Z}} \mathbf{x} \times \mathbf{T} \mathbf{n} d a d t^{\prime},
$$

where $\lambda, \chi, \omega$ are arbitrary scalar-valued functions on $\mathscr{R}$; really, (4.14)-(4.16) hold with independent $\lambda$ 's, $\chi$ 's, and $\omega$ 's for the various components. The particular case when $\lambda, \chi, \omega$ are Dirac's delta function $\delta\left(t^{\prime}\right)$ gives

$$
\rho \overline{\mathbf{u}}(t)=\int_{0}^{t} \overline{\mathbf{F}} d t^{\prime}+\rho \overline{\mathbf{v}}+\frac{1}{V} \int_{0}^{t} \int_{\partial Z^{\prime}} \mathbf{T n} d a d t^{\prime},
$$




$$
\begin{aligned}
& \operatorname{sym}(\overline{\mathbf{x} \otimes \rho \dot{\mathbf{u}}})(t)+\int_{0}^{t} \overline{\mathbf{T}} d t^{\prime}= \int_{0}^{t} \operatorname{sym}(\overline{\mathbf{x} \otimes \mathbf{F}}) d t^{\prime}+\operatorname{sym}(\overline{\mathbf{x} \otimes \rho \mathbf{v}}) \\
&+\frac{1}{V} \int_{0}^{t} \int_{\partial^{\prime} Z} \operatorname{sym}(\mathbf{x} \otimes \mathbf{T n}) d a d t^{\prime}, \\
& \overline{\mathbf{x} \times \rho \dot{\mathbf{u}}}(t)=\int_{0}^{t}(\overline{\mathbf{x} \times \mathbf{F}}) d t^{\prime}+\overline{\mathbf{x} \times \rho \mathbf{v}}+\frac{1}{V} \int_{0}^{t} \int_{\partial^{\prime Z}} \mathbf{x} \times \mathbf{T n} d a d t^{\prime} .
\end{aligned}
$$

Alternatively, we could have obtained (4.17)-(4.19) directly, without having recourse to Dirac's delta function, by considering $\lambda_{k}, \chi_{k l}, \omega_{k l}$ as independent of $t$.

Incidentally, (4.17) and (4.19) are the integral version of balance equations for linear and angular momentum. They give the means $\overline{\rho \dot{\mathbf{u}}}$ and $\overline{\mathbf{x} \times \rho \dot{\mathbf{u}}}$ in terms of the boundary and initial data, namely $\mathbf{T n}$ on $\partial \mathscr{D} \times \mathscr{R}^{+}$and $\mathbf{v}$ on $\mathscr{Z}$, and the known body force $\mathbf{F}$ on $\mathscr{D} \times \mathscr{R}^{+}$. Equation (4.18), instead, is unusual as a balance equation; it provides an estimate on $\operatorname{sym}(\overline{\mathbf{x} \times \rho \dot{\mathbf{u}}})$ or $\overline{\mathbf{T}}$ when information is given on one of the two quantities.

ii) A conservation law involving the displacement. A less usual conservation law follows by considering the case when the density $I$ involves the displacement $\mathbf{u}$, rather than the velocity $\dot{\mathbf{u}}$.

Formally, let $\tilde{w}_{k}=\psi_{k} / p$ and $w_{k}=\mathscr{L}^{-1}\left\{\psi_{k} / p\right\}$. By paralleling, step by step, the previous procedure we have

$$
I=-w_{k} * \rho u_{k}
$$

where, again, $w_{k}$ is given by (4.10). Then from

$$
\frac{\partial \hat{\sigma}_{h}}{\partial x_{h}}=\frac{1}{p} \tilde{w}_{k} \tilde{F}_{k}+\frac{1}{p} \rho \tilde{w}_{k} v_{k}
$$

we have

$$
\frac{\partial \sigma_{h}}{\partial x_{h}}=w_{k} * \mathscr{F}_{k}+\rho v_{k} \mathscr{W}_{k} \text {, }
$$

where $\mathscr{F}_{k}$ and $\mathscr{W}_{k}$ are the integrals, over the time interval, of $F_{k}$ and $w_{k}$. Meanwhile

$$
J_{i}=w_{k} * G_{k i h j} * u_{j h}-\chi_{k h} * G_{k h i j} * u_{j}+\sigma_{i} .
$$

By (3.1) and (4.20), (4.21), integration over $\mathscr{D} \times[0, t)$ yields

$$
\begin{aligned}
\overline{w_{k} * \rho u_{k}}= & \int_{0}^{t} \overline{w_{k} * \mathscr{F}_{k}} d t^{\prime}+\int_{0}^{t} \rho \overline{v_{k} \mathscr{W}_{k}} d t^{\prime} \\
& +\frac{1}{V} \int_{0}^{t} \int_{\mathscr{Z}}\left(w_{k} * G_{k i j h} * u_{j h}-\chi_{k h} * G_{k h i j} * u_{j}\right) n_{i} d a d t^{\prime}
\end{aligned}
$$

which in fact gives the three conservation laws

$$
\begin{aligned}
&(\lambda * \rho \overline{\mathbf{u}})(t)=\int_{0}^{t} \lambda * \overline{\mathscr{F}} d t^{\prime}+\rho \overline{\mathbf{v}} \int_{0}^{t} \int_{0}^{t^{\prime}} \lambda(\tau) d \tau d t^{\prime}+\frac{1}{V} \int_{0}^{t} \int_{\mathscr{Z}} \lambda * \mathbf{n} \mathbf{G} * \nabla \mathbf{u} d a d t^{\prime}, \\
& {[\chi * \operatorname{sym}(\overline{\mathbf{x} \otimes \rho \mathbf{u}})](t)=} \int_{0}^{t}\left[\chi * \operatorname{sym}(\overline{\mathbf{x} \otimes \mathscr{F}}) d t^{\prime}+\operatorname{sym}(\overline{\mathbf{x} \otimes \rho \mathbf{v}}) \int_{0}^{t} \int_{0}^{t^{\prime}} \chi(\tau) d \tau d t^{\prime}\right. \\
&+\frac{1}{V} \int_{0}^{t} \int_{\zeta}[\chi * \operatorname{sym}(\mathbf{x} \otimes \mathbf{n G} * \nabla \mathbf{u})-\chi * \mathbf{G} *(\mathbf{u} \otimes \mathbf{n})] d a d t^{\prime},
\end{aligned}
$$




$$
\begin{aligned}
(\omega * \overline{\mathbf{x} \times \rho \mathbf{u}})(t)= & \int_{0}^{t}(\omega * \overline{\mathbf{x} \times \mathscr{F}}) d t^{\prime}+\overline{\mathbf{x} \times \rho \mathbf{v}} \int_{0}^{t} \int_{0}^{t^{\prime}} \omega(\tau) d \tau d t^{\prime} \\
& +\frac{1}{V} \int_{0}^{t} \int_{\mathscr{Z}} \omega * \mathbf{x} \times(\mathbf{n G} * \nabla \mathbf{u}) d a d t^{\prime}
\end{aligned}
$$

whereby the knowledge of $\mathbf{u}$ and $\nabla \mathbf{u}$ on the boundary $\partial \mathscr{D}$ and of the body force $\mathbf{F}$ on $\mathscr{D} \times[0, t)$ leads at once to estimates on the means $\overline{\mathbf{u}}$ and $\overline{\mathbf{x} \otimes \mathbf{u}}$. As before, if $\lambda, \chi$, and $\omega$ are taken as Dirac's delta functions then convolutions reduce to the current value of the pertinent physical quantity.

iii) Conservation laws related to reciprocity theorems. Every self-adjoint linear system does always possess a reciprocity relation $[1,7,8,16]$ which allows the determination of a conservation law canonically associated with every pair of solutions to the given equations. Conversely, it has been shown in linear elastostatics [3, 17] and in linear elastodynamics [18] that a reciprocal theorem follows from the knowledge of a complete set of conservation laws; it takes the explicit form of a Betti theorem whereby, given two elastic states, the work done by the external forces of the first over the displacements of the second is related to the work done by the external forces of the second over the displacements of the first. We observe that the system (2.8) is linear and self-adjoint, since it arises from the Lagrangian (2.12). Thus, notwithstanding possible difficulties that may occur in connection with the problem of the time-derivative, we expect that a result similar to the Betti's reciprocal theorem holds.

To show this, consider a new viscoelastic state $\left(\mathbf{u}^{\star}, \mathbf{E}^{\star}, \mathbf{T}^{\star}\right)$ corresponding to the same relaxation function $\mathbf{G}$ and denote by a superimposed * the pertinent quantities. Then look at the conservation law (4.1) and choose $k_{i}$ and $\widehat{I}$ as

$$
\begin{gathered}
k_{i}=-\frac{1}{p} \frac{\partial \psi_{k}}{\partial x_{k}} \widetilde{G}_{k h i l} \tilde{u}_{l}, \\
\widehat{I}=-\frac{1}{p}\left[\psi_{k}\left(\rho \tilde{u}_{k}-\tilde{f}_{k}\right)+\tilde{u}_{k}\left(\rho \psi_{k}-\tilde{f}_{k}^{\star}\right)\right],
\end{gathered}
$$

where of course $\tilde{f}_{k}^{\star}$ is given by the counterpart of Eq. (2.10), and let $I_{0}=0$. In view of (4.23) and (4.24), (4.1) becomes

$$
-\frac{1}{p} \frac{\partial^{2} \psi_{k}}{\partial x_{h} \partial x_{i}} \widetilde{G}_{k h i l} \tilde{u}_{l}+\tilde{u}_{l}\left(\rho \psi_{l}-\tilde{f}_{l}^{\star}\right)=0
$$

which is satisfied whenever $\psi_{l}$ coincides with the solution $\tilde{u}_{l}^{\star}$ to the counterpart of (2.8). Hence the explicit expressions for $k_{i}$ and $\hat{I}$ become

$$
\begin{gathered}
k_{i}=-\frac{1}{p} \frac{\partial \tilde{u}_{k}^{*}}{\partial x_{h}} \tilde{G}_{k h i l} \tilde{u}_{l}, \\
\widehat{I}=-\frac{1}{p} \tilde{u}_{k}^{\star}\left(\rho \tilde{u}_{k}-\tilde{f}_{k}\right)+\frac{1}{p} \tilde{u}_{k}\left(\rho \tilde{u}_{k}^{\star}-\tilde{f}_{k}^{*}\right) .
\end{gathered}
$$

The application of the inverse Laplace transform shows that $(4.26)$ is consistent with the choice $I_{0}=0$ and provides the following result. 
Let $(\mathbf{u}, \mathbf{E}, \mathbf{T})$ and $\left(\mathbf{u}^{\star}, \mathbf{E}^{\star}, \mathbf{T}^{\star}\right)$ be viscoelastic states on $\overline{\mathscr{D}} \times(0, \infty)$ corresponding to the same relaxation function $\mathbf{G}$, as in $(2.1)$, to the body forces $\mathbf{F}$ and $\mathbf{F}^{\star}$, to the initial data $\mathbf{d}, \mathbf{v}$ and $\mathbf{d}^{\star}, \mathbf{v}^{\star}$, respectively. Then, for any $t \in(0, \infty)$, the conservation law (3.1) holds with

$$
\begin{gathered}
J_{i}=\int_{0}^{t}\left(u_{k}^{\star} * G_{k i h j} * u_{j h}-u_{h k}^{\star} * G_{k h i l} * u_{l}\right)\left(t^{\prime}\right) d t^{\prime}, \\
I=\int_{0}^{t}\left\{-u_{k} *\left[g * F_{k}^{\star}+\rho\left(g v_{k}^{\star}+d_{k}^{\star}\right)\right]+u_{k}^{\star} *\left[g * F_{k}+\rho\left(g v_{k}+d_{k}\right)\right]\right\}\left(t^{\prime}\right) d t^{\prime},
\end{gathered}
$$

where $\mathbf{u}^{\star}$ is the solution to the problem $(2.3),(2.4)$ with body force $\mathbf{F}^{\star}$ and initial data $\mathbf{d}^{\star}$ and $\mathbf{v}^{\star}$.

5. The connection with Noether's theorem. Owing to the application of Noether's theorem for the derivation of conservation laws it is worth investigating the possible connection between divergence-free fields and Noetherian fields, i.e., conserved vectors that are generated via Noether's approach. This connection would have the advantage of providing divergence-free fields with the physical meaning usually associated with Noetherian fields in terms of the generators of infinitesimal invariance transformations [1, 4, 18].

Since the Lagrangian for the viscoelastic body is provided by (2.12) we can apply the Noether-type approach developed in $[12,18]$ whereby

$$
\widehat{L} D_{i} \hat{h}_{i}+\frac{\partial \widehat{L}}{\partial x_{i}} \hat{h}_{i}+\frac{\partial \widehat{L}_{i}}{\partial \tilde{u}_{i}}+\frac{\partial \widehat{L}}{\partial \tilde{u}_{i j}}\left(D_{j} \hat{\xi}_{i}-\tilde{u}_{i k} D_{j} \hat{h}_{k}\right)-D_{i} \widehat{M}_{i}+p \widehat{I}-I_{0} \doteq 0
$$

is the conservation law which arises from the invariance of the functional $\int_{y} \widehat{L} d V$ under the infinitesimal transformation

$$
\begin{aligned}
& x_{i} \mapsto x_{i}+\varepsilon \hat{h}_{i}(\mathbf{x}, \tilde{\mathbf{u}}, \nabla \tilde{\mathbf{u}}), \\
& \tilde{u}_{i} \mapsto \tilde{u}_{i}+\varepsilon \hat{\xi}_{i}(\mathbf{x}, \tilde{\mathbf{u}}, \nabla \tilde{\mathbf{u}}),
\end{aligned}
$$

where $\varepsilon$ denotes a "small" parameter and $\hat{h}_{i}$ and $\hat{\xi}_{i}$ are the generators of the invariance transformation. Here $\widehat{M}_{i}$ denotes an arbitrary set of functions of $\mathbf{x}, \tilde{\mathbf{u}}, \nabla \tilde{\mathbf{u}}$ arising from the fact that the invariance of the functional is meant up to a divergence term. Upon use of the Euler-Lagrange equations

$$
\frac{\partial \widehat{L}}{\partial \tilde{u}_{i}}-D_{j} \frac{\partial \widehat{L}}{\partial \tilde{u}_{i j}}=0
$$

we can write $(6.1)$ as

$$
D_{i} \widehat{J}_{i}+p \widehat{I}-I_{0} \doteq 0
$$

with

$$
\widehat{J}_{i}=\widehat{L} \hat{h}_{i}+\frac{\partial \widehat{L}}{\partial \tilde{u}_{i j}}\left(\hat{\xi}_{j}-\tilde{u}_{j k} \hat{h}_{k}\right)-\widehat{M}_{i} .
$$

This establishes a formal equivalence between the two approaches, the correspondence being specified by (5.2). Operatively, the search for the general solution to (5.1) is usually more involved and then, often, appropriate restrictions on the form of the 
unknown functions are introduced. For instance, in [12] we solved the problem by letting $\hat{h}_{i}=\hat{h}_{i}(\mathbf{x}, \tilde{\mathbf{u}}), \hat{\xi}_{i}=\hat{\xi}_{i}(\mathbf{x}, \tilde{\mathbf{u}})$ and $\widehat{M}_{i}$ be linear in $\nabla \tilde{\mathbf{u}}$. Here, instead, we have found the general form of the dependence of $\widehat{J}_{i}$ on the derivatives $\tilde{u}_{i j}$, showing in particular that $\widehat{J}_{i}$ is necessarily quadratic.

In conclusion, there seems to be technical advantages in searching for conservation laws through the divergence form (3.2).

6. Derivation of conserved fields in elasticity. Besides proceeding along the same guidelines, the search for conservation laws in elasticity develops through quite similar conditions. The constitutive equation (analog of $(2.1)$ ) is

$$
\mathbf{T}(\mathbf{x}, t)=\mathbf{C E}(\mathbf{x}, t),
$$

with $\mathbf{C} \in \mathbf{L}($ Lin, Lin) being constant and satisfying the symmetry conditions (2.2). Then the equations of motion (2.3) ultimately become (cf. (2.8))

$$
\rho \tilde{u}_{i}=\frac{1}{p^{2}} C_{i j k l} \tilde{u}_{k l j}+\tilde{f}_{i}
$$

$\tilde{f}_{i}$ being the same as in (2.10). The Lagrangian $\widehat{L}$ is given by

$$
\widehat{L}=\frac{1}{2 p^{2}} C_{i j k l} \tilde{u}_{i j} \tilde{u}_{k l}-\tilde{f}_{i} \tilde{u}_{i}+\frac{1}{2} \rho \tilde{u}_{i} \tilde{u}_{i} .
$$

This shows that, in connection with the Laplace transform, the equations for elasticity are put in 1-1 correspondence with those for viscoelasticity by simply letting C correspond to $p \widetilde{\mathbf{G}}$.

Of course, the correspondence of $\mathbf{C}$ with $p \widetilde{\mathbf{G}}$, rather than $\widetilde{\mathbf{G}}$ itself, leads to quite different time dependences when the inverse Laplace transformation is applied. This difference is hardly surprising from a mathematical viewpoint. As we expect it to be, important differences arise as to the mechanical behaviour associated with the corresponding conservation laws. This feature will be the subject of a future investigation.

Acknowledgments. The research leading to this work has been performed under the auspices of the National Group for Mathematical Physics of C.N.R. and partially supported by the Italian Ministry of Education through the Research Project "Problemi di evoluzione nei fluidi e nei solidi."

\section{REFERENCES}

[1] P. J. Olver, Applications of Lie Groups to Differential Equations, Springer, New York, 1986

[2] F. Bampi and A. Morro, The inverse problem of the calculus of variations applied to continuum physics, J. Math. Phys. 23, 2312-2321 (1982)

[3] P. J. Olver, Conservation law's in elasticity. I. General results, Arch. Rational Mech. Anal. 85, $112-129(1984)$

[4] D. G. Edelen, Applied Exterior Calculus, Wiley, New York, 1985

[5] D. G. B. Edelen and I. M. Snyman, Cartan forms for multiple integral problems in the calculus of variations, J. Math. Anal. Appl. 120, 218-239 (1986)

[6] G. Caviglia and A. Morro, Noether-type conservation laws for perfect fluid motions, J. Math. Phys. 28, $1056-1060$ (1987) 
[7] G. Caviglia, Symmetry transformations, isovectors, and conservation laws, J. Math. Phys. 27, 972978 (1986)

[8] G. Caviglia, Composite variational principles and the determination of conservation laws, J. Math. Phys. 29, 812-816 (1988)

[9] R. Arens, The conserved currents for the Maxwellian field, Commun. Math. Phys. 90, 527-544 (1983)

[10] M. J. Leitman and G. M. C. Fisher, The linear theory of viscoelasticity, Encyclopedia of Physics. Vol. VI A/3, Springer, Berlin, 1973, pp. 1-123

[11] Q. Jiang, Conservation laws in linear viscoelastodynamics, J. Elasticity 16, 213-219 (1986)

[12] G. Caviglia and A. Morro, A general approach to conservation law's in viscoelasticity, Acta Mech. 75, 255-267 (1988)

[13] V. I. Fushchich and A. G. Nikitin, New and old symmetries of the Maxwell and Dirac equations. Sov. J. Part. Nucl. 14, 1-22 (1983)

[14] V. I. Fushchich and A. G. Nikitin, Symmetries of Maxwell's Equations, Reidel, Dordrecht, 1987

[15] M. E. Gurtin and E. Sternberg, On the linear theory of viscoelasticity. Arch. Rational Mech. Anal. 11, 291-356 (1963)

[16] P. J. Olver, Conservation Law's in Elasticity. III. Planar Linear Anisotropic Elastostatics, Arch. Rational Mech. Anal. 102, 167-181 (1988)

[17] G. Caviglia and A. Morro, Conservation laws in anisotropic elastostatics, Int. J. Engrg. Sci. 26. 393-400 (1988)

[18] G. Caviglia and A. Morro, Generalized symmetries and conservation laws: a Noether-type approach. Riv. Mat. Pura Appl. 4, 33-54 (1989) 